\title{
The sustainable architectural design of post-disaster reconstruction of the aboriginal settlements in Taiwan
}

\author{
C.-J. Chen \\ Department of Creative Design and Architecture, \\ National University of Kaohsiung, Taiwan
}

\begin{abstract}
Due to the influence of global change, the frequency of natural disasters in Taiwan has risen in recent years. Typhoon Morakot wrought catastrophic damage in August 2009, leaving 461 people dead and 192 others missing, most of whom are feared dead. In addition to roughly \$3.3 billion USD accrued in damages, many human settlements were destroyed and inhabitants were forced to leave their homeland; this was especially true for the indigenous aboriginal people.

Various effective measures were immediately mobilized by the Post-Disaster Reconstruction Council. Different community reconstruction plans were eventually organized, which focused on rebuilding living spaces and related facilities for the affected inhabitants. Although various architectural programs were carried out to establish temporary settlements on new sites for impacted communities, the new buildings are somehow considered unacceptable or inappropriate by inhabitants.

The focus of this research is to analyze the sustainability of reconstructing aboriginal settlements and communities in Taiwan. The goal of this research is to systematically induce the key problems by means of case study. Not only are site selection, environmental assessment, and building type discussed, but the influence of different socio-economic and cultural issues are also considered. The sustainable considerations of architectural design are concluded as a result of this research. More appropriate design concepts will also be proposed to integrate into further post-disaster reconstruction programs.

Keywords: post-disaster, reconstruction plans, architectural design, aboriginal settlement.
\end{abstract}




\section{Introduction}

Global changes are responsible for more unforeseen natural disasters in recent years in Taiwan. Post-disaster reconstruction has thus become Taiwan's focus. For residents greatly affected by the disaster, not only are their estates and properties damaged or lost, but their psychological and physiological status is seriously harmed. In accordance with past experiences, the primary intervention mechanism of post-disaster reconstruction is to move and replace the affected residents to a safer location. Another important and immediate measure is to establish emergency or temporary shelters. Both interventions satisfy the physical needs of affected people. The resettlement policy that disregards different cultural backgrounds of various ethnic groups usually is another contributor of damage. This phenomenon most commonly occurs to the aboriginal settlements since they still live in the most environmentally sensitive areas in Taiwan. This research will focus on the sustainability of reconstructing aboriginal settlements and communities. The more appropriate environmental planning principles as well as architectural design concepts will be proposed.

\section{Purpose of research}

Hasty governmental decisions in post-disaster rehabilitation sometimes forces affected residents to deviate from their original habitats, resulting in negative consequences during reconstruction. What constitutes appropriate considerations regarding the integration of different cultures and lifestyles in post-disaster reconstruction programs seems to be the most important task.

Regardless of whether a program originates from a governmental or nongovernmental organization, past models are to be followed for the construction of permanent housing models or selection of land. Most of the projects maintain an "easy to operate" logic, which ignores the real needs of residents. To operate with regard to different regions, different ethnic groups, and different cultural backgrounds for different regions, ethnic groups should be emphasized in the future.

The purpose of this study is to:

(1) systematically induce the key problems by means of case study;

(2) identify the planning and design principles concerning site selection, environmental assessments, and design concepts.

\section{Methodology of research}

EEWH established in 1999, is the green building certification system in Taiwan. It comprises nine indicators that fall into four categories - ecology, energy saving, waste reduction and health. EEWH is also used as basis of the other four specific evaluation systems, they are: EEWH-EC (Ecological Community, 2009), EEWH-GF (Green Factory, 2010), EEWH-RN (Renovation, 2010) and EEWH-RS (Residential, 2011). The EEWH-EC evaluation system is aimed at Taiwanese laws, cultural background, social conditions and characteristics of the 
community development out of the system, all of the assessment of the content is mostly within the context of jurisdiction of architecture and urban policy, extending the ecology, energy conservation and waste reduction, health and comfort from the EEWH-Green Building Rating System, together with service function and crime prevention, which are community-oriented. Figure 1 shows the structure of the EEWH-Eco Community [1].

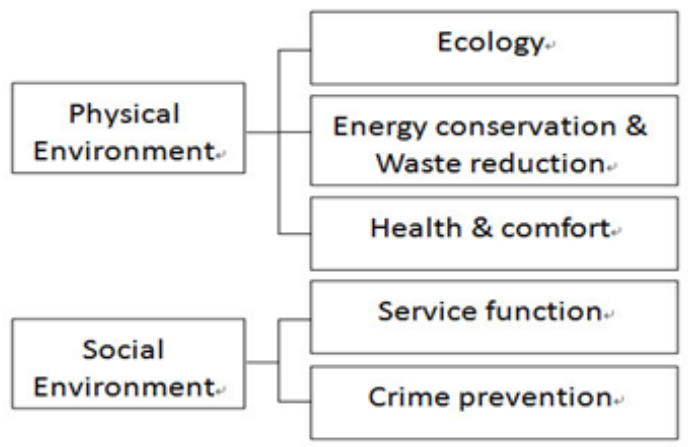

Figure 1: $\quad$ Structure of the EEWH-Eco community.

Although various sustainable design criteria based on EEWH of the modern architecture are well defined in Taiwan since 1999, nevertheless, the different subjects between vernacular architecture and modern architecture should be clarified through in field investigation.

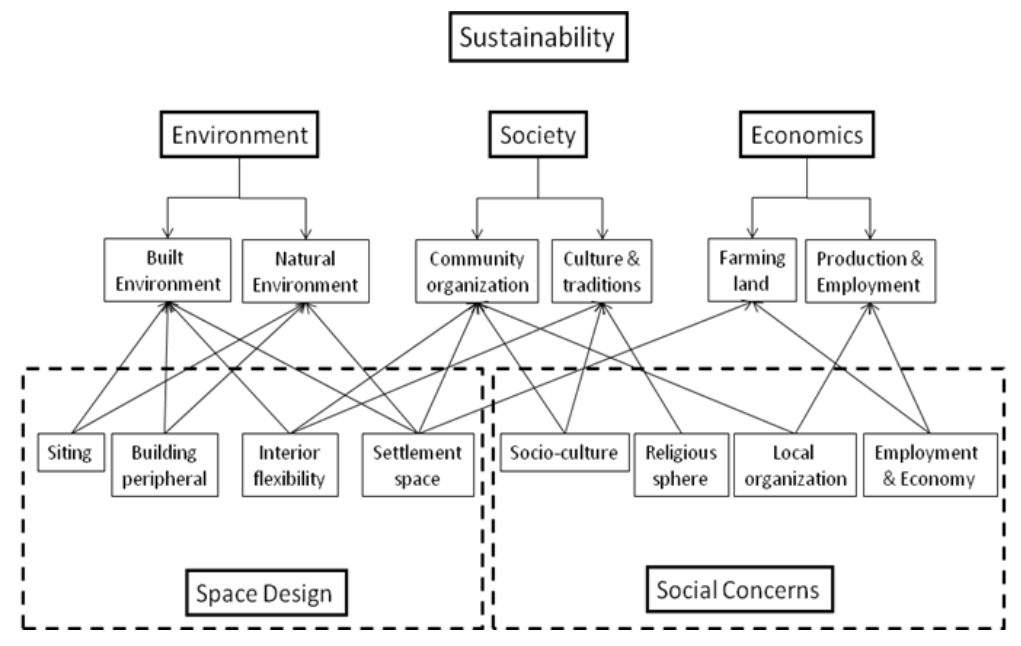

Figure 2: $\quad$ Two considerations and sustainability of human settlement. 
EEWH mainly focus on physical environment rather than on social environment, which could not response the prompt demands of affected residents. Hence, more suitable considerations are proposed in this study regarding site suitability, social life, and various interviews in field investigation. Two major considerations containing eight items are proposed. Space design and social concerns are the two main considerations. Figure 2 explains the connection between sustainability and the detailed items.

Space design consists of four items: siting, building peripherals, interior flexibility, and settlement space configuration. Details of each item considered are as follows:

(1) Siting is necessary to compare the traditional and in-being living environments. The difference can be thus defined when making decisions about a new site.

(2) Building peripherals including cultivated land, a cemetery, and public space are discussed to ensure the living functions.

(3) Interior flexibility includes reasonable space order and arrangement, but also the possibility of rearrangement and the physical quality of a building are considered.

(4) Settlement space configurations reflect the different ethnic groups' own social and cultural orders. The space arrangement of settlement refers to both cultural identification and the inheritance of society.

Social concerns contain four items: socio-culture, employment and economics, local organization, and the religious sphere.

(1) Socio-culture refers to the idea that every ethnic group is able to create their own decorative vocabulary, such as indigenous totems, traditional carvings, and artifacts. A new environment must allow the succession of creative potentiality.

(2) Employment and economy refers to the notion that a new living location has to provide accessible connections to employment and offer the possibility of economical developments that guarantee the sustainability of tribes.

(3) Local organization of the settlements is usually represented by both traditional organization (tribal assembly) and new administrative institutions. Both organizations should elaborate in parallel.

(4) The reconstruction program should take into consideration different religious backgrounds of different ethnic backgrounds. Moreover, a spiritual square and worship place are also required.

The importance of the aforementioned items is specified later through field investigation interviews with affected residents.

\section{Case study}

\subsection{Settlement}

The selected tribe of this is study is the Rukai, which is the fifth largest indigenous group living in the mountains of southern Taiwan. The traditional home of the Rukai tribe is considered to be Kochapongane, also known as Old 
Haocha. Old Haocha is situated on the contour of mountains. The altitude of the settlement is around 900 to 1000 meters, where the relative mild climate provides both rich hunting resources as well as fertile soil. The houses of the village were built on a virtually linear path along the contour (see Figure 3). Though the original village of Old Haocha maintains a significant collection of traditional slate homes of many legendary places, the village people were moved to new territories twice due to modernization and natural disasters (see Figure 4). The last migration was due to Typhoon Morakot in 2009. The village's name, "Rinari,” (see Figure 5) was jointly decided by all residents [2].

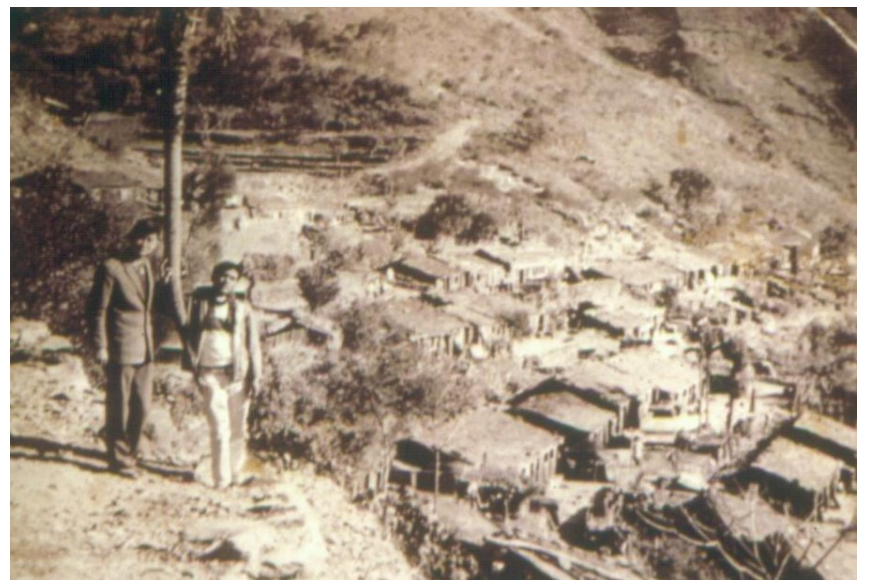

Figure 3: $\quad$ Original Old Haocha Village (before 1978).

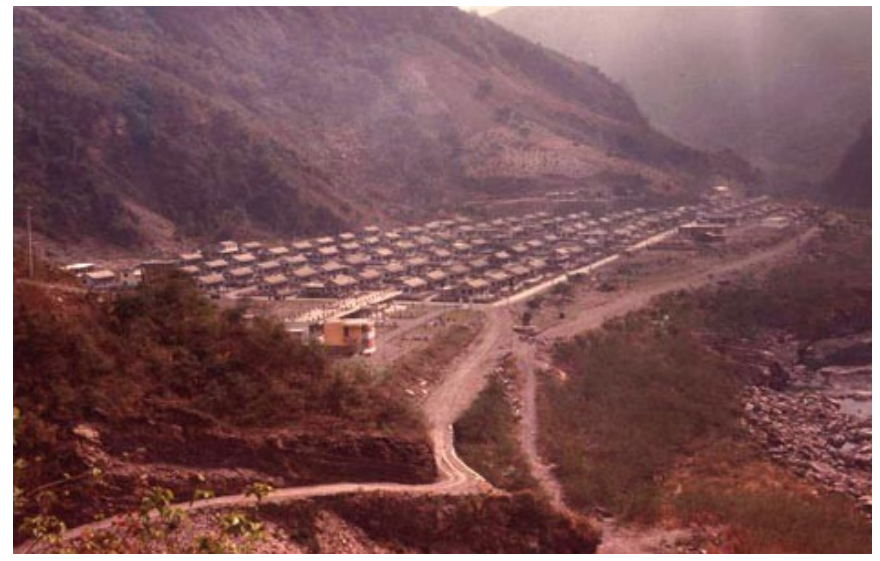

Figure 4: $\quad$ First Resettled Village of New Haocha (1978-2009). 


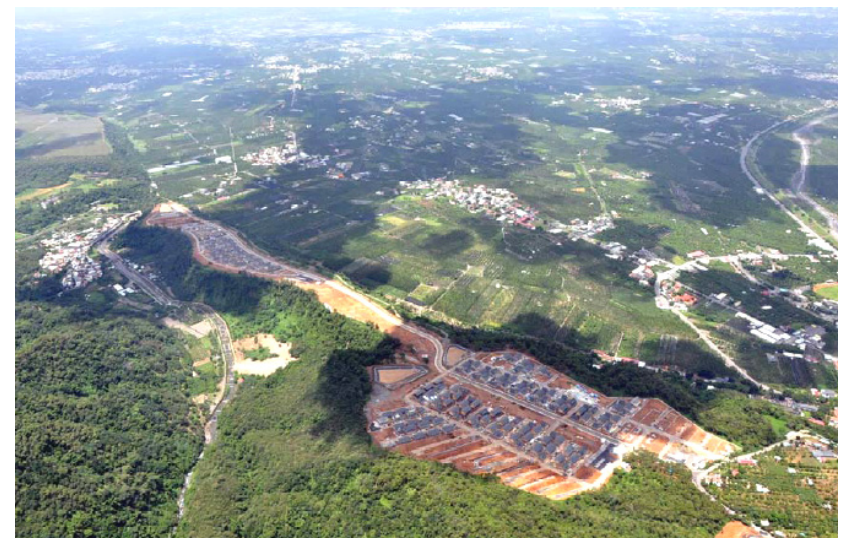

Figure 5: A bird's-eye view of Rinari permanent housing base. (http://start.org/download/2012/ai-irdr/4-2-renari.pdf) [3].

The Rinari permanent housing base on the Maja farm covers an area of 27.8 hectares, and is situated in the Ailiao section, Neipu Township in Pingtung County. World Vision Taiwan helped build 483 units (1,152 square feet) of permanent housing on the land, with the government and World Vision Taiwan share expenditures resulting from the public facilities in the base. The Rinari permanent housing base aims to settle 336 flood-stricken families from the Rukai tribe of Haocha Village.

\subsection{Construction features}

In order to represent the indigenous cultures, history, and diversified life experiences, a number of facilities are built. These include an image design of the tribe entrance and the "story wall," three comprehensive activity centers, children recreation rooms, a leisure square, a sports court, a youth club, a tribal reconstruction pavilion, and a sales shop. Instead of standardized housing, these facilities take into account the socio-cultural concern of tribes.

The standardized houses were built in modern ways. Light-weight steel structures with a wooden shingle appearance possess a total surface of $130 \mathrm{~m}^{2}$ on two floors. These houses (see Figure 6) are considered simple, sufficient, and contemporary, but nevertheless, they do not reflect any traditional patterns, as shown in stone slate houses of the old village (see Figure 7). The most common phenomenon is that once residents move in, they begin to immediately modify their own façade decorations with old totems, and erect the front garden or court with stone slate with traditional features (see Figures 8 and 9). The participatory design, as well as self reliance, is considered an important expression of confidence of tribal culture. 


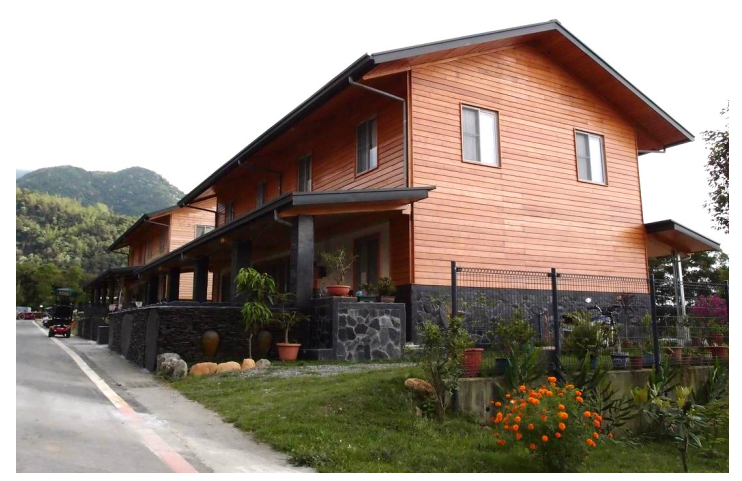

Figure 6: $\quad$ Permanent house in Rinari.

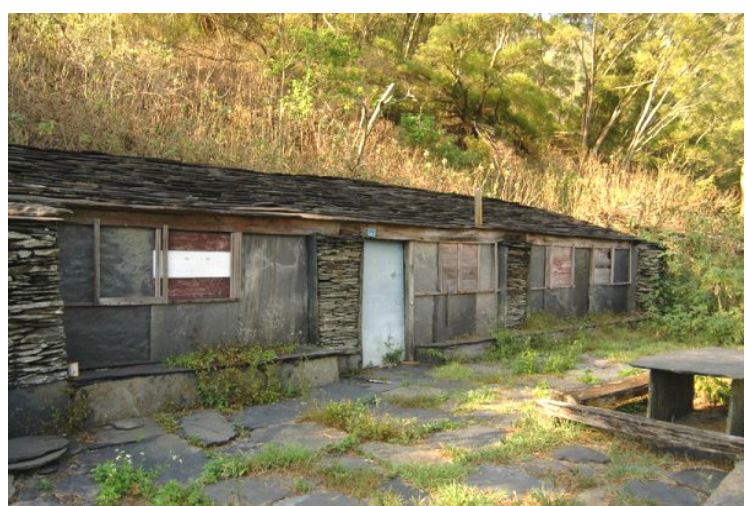

Figure 7: Traditional slate house of Old Haocha (source: http://starryalley.twbbs.org/gallery2/d/18516-2/dsc 5094.jpg).

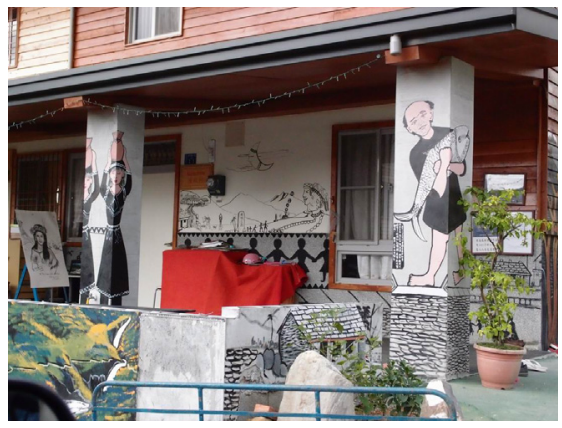

Figure 8: Decorative façade.

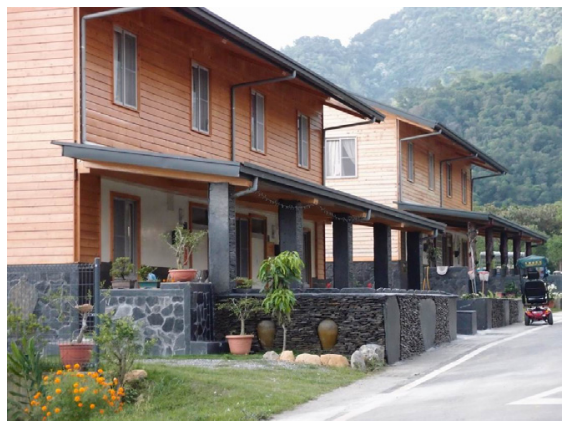

Figure 9: Front court. 


\subsection{Comparison of building types}

The traditional house for the Rukai tribe, shown in Figure 10, is a low-rise slate construction. The separation of inner space appeared from the Japanese occupation period (1895-1945). Every house unit had its independent territory: space, granary, and courtyard, forming a self-sufficient habitat system [4].
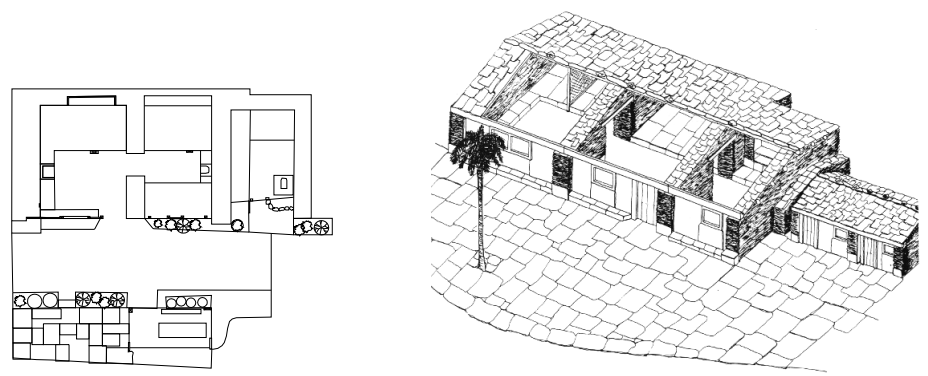

Figure 10: Traditional slate house of the Rukai Tribe (before 1970).

In order to improve the tribe's living conditions, a governmental program resettled the Old Haocha to a new site, called New Haocha during the 1970s. The standard building type, as shown in Figure 11, covers a total surface of $132 \mathrm{~m}^{2}$. All houses are regularly aligned along the roads. Both housing type and settlement arrangement maintain the same characteristics present in Old Haocha.
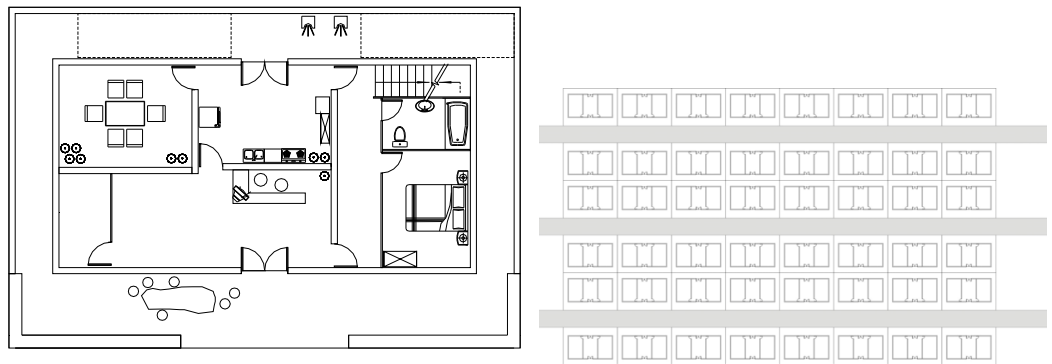

Figure 11: Modified house (left) aligned along the roads (right) in New Haocha.

In Rinari, although the "Full Recovery" concept was emphasized as the core task of the reconstruction program, some elements of the newly built environment are considered insufficient and incomplete. For example, new buildings impose the value of modern society without thinking of space identification of the tribe itself. People value inspiration from the traditional atmosphere that is created by their ancestors more than the new material and structure (Figure 12). 

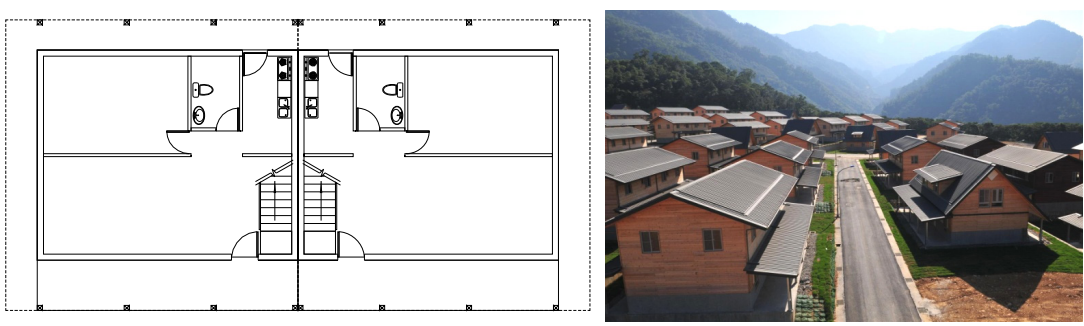

Figure 12: Configuration of a new house in Rinari.

\section{Results and conclusions}

Thirty-nine subjects were interviewed. The results of the priority of proposed items for two major conditions, respectively, are displayed in Table 1.

Table 1: $\quad$ Results of Interview.

\begin{tabular}{|c|c|c|}
\hline \multicolumn{1}{|c|}{ Considerations } & Items & Priority \\
\hline \multirow{4}{*}{ Space Design } & Settlement space & 1 \\
\cline { 2 - 3 } & Building peripheral & 2 \\
\cline { 2 - 3 } & Siting & 3 \\
\cline { 2 - 3 } Social Concerns & Interior flexibility & 4 \\
\cline { 2 - 3 } & Socio-culture & 1 \\
\cline { 2 - 3 } & Employment and economics & 2 \\
\cline { 2 - 3 } & Local organization & 3 \\
\hline
\end{tabular}

Combining this result with the configurations of settlements during different periods, we can figure out the real demands from affected residents. The core cultural value for the Rukai people is coexisting between human and nature, the link between present and tradition. The rehabilitation and reconstruction program focus more comprehensively on both psychological and physiological considerations. When it comes to building design, people prefer to have more flexibility to account for gradual changes in living conditions and needs. Mankind should not be arranged by formal space without paying attention to the operations of a home. The reversibility of the structural system and space should be taken into account for further design.

\section{References}

[1] EEWH Assessment System for Eco-community, About Green Building Labeling, Question and Answers (http://twgbqanda.com/english/).

[2] Brief Description of Rinari (cms-88flood.www.gov.tw).

[3] Rinari Permanent Housing Base, National Science and Technology Center for Disaster Reduction, 2010.

[4] Chijiiwa Suketarou, The Dwellings of Aborigines in Taiwan (in Japanese), 1960 Tokyo, 1988 Taipei. 